\title{
Toxin-mediated infectious botulism
}

INSERM

\section{Source}

INSERM. (1999). Orphanet: an online rare disease and orphan drug data base. Toxinmediated infectious botulism. ORPHA:230800

Infectious botulism is a form of botulism (see this term), a rare acquired neuromuscular junction disease, characterized by descending flaccid paralysis caused by botulinum neurotoxins (BoNTs), produced in vivo leading to toxin-mediated infection. Infectious botulism includes wound botulism and intestinal toxemia botulism (infant botulism and adult intestinal botulism; see these terms). 